
\title{
25 Research Square \\ Low Urine Secretion of Semaphorin3A In Lupus Patients With Proteinuria
}

Doron Rimar ( $\nabla$ doronrimar@gmail.com )

Bnai Zion Medical Center https://orcid.org/0000-0002-9137-2322

Merav Lidar

Sheba Medical Center at Tel Hashomer: Sheba Medical Center

Nasrin Eiza

Bnei Zion Hospital

Adi D Sabag

Bnei Zion Hospital

Elias Toubi

Bnei Zion Hospital

Gleb Slobodin

Bnei Zion Hospital

Itzhak Rosner

Bnei Zion Hospital

Michael Rozenbaum

Bnei Zion Hospital

Zahava Vadasz

Bnei Zion Hospital

\section{Research Article}

Keywords: Semaphorin3A, VEGF, SLE, Lupus nephritis

Posted Date: August 6th, 2021

DOl: https://doi.org/10.21203/rs.3.rs-750580/v1

License: (c) (i) This work is licensed under a Creative Commons Attribution 4.0 International License.

Read Full License 


\section{Abstract}

Background: Immune semaphorins are important players in controlling both innate and adaptive immune responses. The regulatory role of semaphorin3A (sema3A) in systemic lupus erythematosus (SLE), rheumatoid arthritis (RA) and other autoimmune diseases is widely reported. Decreased levels of serum sema3A were shown to be associated with SLE disease activity.

Objectives: To assess urine concentrations of sema3A in SLE patients and its correlation with renal involvement and disease activity.

Methods: Urine levels of sema3A were analyzed in 38 SLE patients of whom 13 had renal involvement and were compared to 10 healthy controls and 8 RA patients (disease control group).

Results: The secretion of urine sema3A was found to be significantly lower in SLE patients compared to healthy controls and RA patients $(4.9 \pm 3.9 \mathrm{ng} / \mathrm{ml}, 8.5 \pm 2.7 \mathrm{ng} / \mathrm{ml}, 9.85 \pm 1.7 \mathrm{ng} / \mathrm{ml}$, respectively, $p=0.0006)$. Urine sema3A was significantly lower in SLE patients with lupus nephritis than in patients without nephritis $(4.0 \pm 3.4 \mathrm{ng} / \mathrm{ml}$ vs $6.5 \pm 3.8 \mathrm{ng} / \mathrm{ml}, \mathrm{p}=0.03)$. Urine sema3A was inversely correlated with proteinuria and SLE disease activity.

Conclusion: Urine sema3A is decreased in lupus patients and should be further evaluated as a possible biomarker for disease activity and renal involvement.

\section{Introduction}

Semaphorins are a large family of proteins, that were initially reported to be involved in axon-guidance, malignancy spread and angiogenesis. Some of these, mainly those who belong to a class of secretory proteins, such as semaphorin3A (sema3A) were recognized as "immune semaphorins" and appreciated to be important players in regulating both innate and adaptive immune responses $(1,2)$. Sema3A was shown to be a potent regulator of CD4 + T cell function, suppressing pro-inflammatory secretion and proliferation of T cells in normal homeostasis and in autoimmune diseases such as in rheumatoid arthritis (RA) (3). Furthermore, sema3A was shown to be a specific marker of T regulatory (Treg) cells, contributing to their normal functional properties. The incubation of recombinant sema3A with Treg cells was reported in many studies to improve functional status of Treg cells and the production of IL-10 by these cells (4). In this respect, we have reported decreased serum levels of sema3A in systemic lupus erythematosus (SLE) compared to normal individuals and in correlation with SLE disease activity (5). Based on these findings, we assessed the benefit of injecting recombinant sema3A to NZB/W mice and were able to demonstrate that this therapy improved SLE-like symptoms in these mice, survival and decreased proteinuria (6). Sema3A is found in the serum as well as other organ tissues including the kidneys, in which sema3A was found to be expressed and secreted by renal podocytes and epithelial cells in distal tubules in kidneys of wild mice models. Though pathophysiology was not fully defined, secreted sema3A seems to be crucial for podocyte survival and for the integrity and function of the kidney 
glomerular filtrates (7). Urine secretion was reported to be significantly increased in animal models with diabetic nephropathy and was found to be correlated with the extent of proteinuria, and renal dysfunction (8). In a recent study, urinary sema3A levels were suggested to be a possible predictive marker in the development of contrast-induced acute renal injury. Serial urine samples of sema3A were obtained at baseline and at intervals of 4 hours following percutaneous coronary intervention (PCI). Increased urine sema3A levels at 2 and 6 hours, post-PCl significantly predicted renal injury (9). The renal pathologies in which sema3A was evaluated were all related to a direct renal injury or persistent ischemia but not to immune-mediated injury. The role of sema3A in the immune mediated renal injury of lupus nephritis has not been evaluated before. This study was designed, aiming to assess urine sema3A secretion in patients with SLE with and without renal involvement and to correlate it with SLE clinical characteristics. The importance of this research lies in the need for new biomarkers, which will, hopefully, promote early diagnosis and better treatment regimens according to the extent of kidney damage.

\section{Patients And Materials}

Patients: Urine was collected from thirty-eight SLE patients (35 women and 3 men; mean age $43 \pm 5$ years). Patients were divided into two groups: The first included 25 patients suffering from arthritis, skin and hematological involvements, but with no renal involvement. The second group included 13 patients suffering from multisystem involvement including renal involvement, of whom seven patients with active nephritis class IV with more than 0.5 gram proteinuria per $24 \mathrm{~h}$ and active sediment and six patients (3 with class IV nephritis and 3 with class $V$ nephritis), who were treated and achieved complete or Partial renal response, defined as at least $50 \%$ reduction in UPCR, normal or near-normal (within $10 \%$ if initially abnormal) GFR and less than $800 \mathrm{mg}$ proteinuria per $24 \mathrm{~h}$ with non-active urine sediment. Table 1 describes the treatments patients received at the time urine was collected. Of notice, although $92 \%$ of patients received prednisone the mean dose was $5 \mathrm{mg} \pm 5.4$. Urine was also collected from 8 patients with rheumatoid arthritis (disease control) and 10 normal individuals, age and gender matched who served as another control group. 
Table 1 describes the treatments patients received at the time urine was collected.

\begin{tabular}{|c|c|c|c|c|}
\hline & $\begin{array}{l}\text { SLE } \\
(n=38)\end{array}$ & $\begin{array}{l}\text { RA } \\
(n=8)\end{array}$ & $\begin{array}{l}\text { Healthy controls } \\
(n=10)\end{array}$ & \\
\hline Age (years) & $59( \pm 18)$ & $51( \pm 19)$ & $52( \pm 15)$ & 0.75 \\
\hline Female (\%) & $33(87 \%)$ & $6(75 \%)$ & $8(80 \%)$ & 0.67 \\
\hline Active nephritis & $7(18 \%)$ & & & \\
\hline Partial renal response * & $6(16 \%)$ & & & \\
\hline APLA & 13(34\%) & & & \\
\hline SLEDAI $2 \mathrm{~K}$ & $8.2 \pm 7.7$ & & & \\
\hline \multicolumn{5}{|l|}{ Treatments } \\
\hline prednisone & $35(92 \%)$ & & & \\
\hline hydroxychloroquine & $25(66 \%)$ & & & \\
\hline methotrexate & $6(16 \%)$ & & & \\
\hline azathioprine & $10(26 \%)$ & & & \\
\hline belimumab & $12(31 \%)$ & & & \\
\hline Mycophenolate mofetil & $8(21 \%)$ & & & \\
\hline rituximab & $6(16 \%)$ & & & \\
\hline abatacept & $2(5 \%)$ & & & \\
\hline IVIG & $5 \%$ & & & \\
\hline \multicolumn{5}{|c|}{$\begin{array}{l}\text { APLA-antiphospholipid antibodies; SLEDAI- systemic lupus erythematosus disease activity score; } \\
\text { IVIG- intravenous immunoglobulins. *Partial renal response- at least } 50 \% \text { reduction in UPCR and } \\
\text { normal or near-normal (within } 10 \% \text { if initially abnormal) GFR and less than } 800 \text { mg proteinuria per } 24 \\
\text { h with non-active urine sediment. }\end{array}$} \\
\hline
\end{tabular}

\section{Methods}

Demographic characteristics and clinical manifestations of lupus patients (i.e. age, gender, weight, height, smoking history, the presence of pleuritic pain, fatigue, night sweats), were assessed by frontal interview and by physical examination (i.e. temperature, alopecia, skin involvement, mucosal ulcers, articular and neurological pathologies). Medical records were examined for: disease onset, kidney involvement, past history of vasculitis, arthritis, pericarditis, and CNS involvement. Laboratory results included: complete blood count, serum creatinine, C3 and C4, autoantibodies (anti-nuclear antibodies-ANA, anti-dsDNA, anticardiolipin IgM and IgG), and 24-hour urine collection for protein. Disease activity was determined for each patient by the SLEDAI 2K score (10). The study was approved by the local Research Ethics Board and all patients gave their informed consent. 


\section{Semaphorin3A urine analyses}

Fifty milliliters of fresh urine samples were collected from all studied individuals. Urine was centrifuged

and the supernatant was then concentrated up to 50 times the initial concentration. Subsequently, all concentrated samples were stored at -20 until analyzed. Semaphorin3A was assessed using a specific commercial human Sema3A ELISA kit (MBS732622, San Diego, CA, USA).

\section{Statistical analysis}

Continuous data are presented as the mean \pm SD. Categorical variables are presented as frequencies and percentages. Comparisons of sema3A levels between categories were made using 2-tailed t-tests. In order to determine the difference between the different patient groups a 1-Way ANOVA and Post hoc tests using Tukey's procedure were performed. We have further evaluated relationships between sema3 $\mathrm{A}$ and disease-related covariates by a correlation test, that is reported via Pearson's $r$. A two-tailed p-value of 0.05 or less was statistically significant. Statistical analysis was performed using the R software (The R Foundation for Statistical Computing 3.0.2 2013).

\section{Results}

Thirty-eight patients suffering from SLE and fulfilling the 2012 SLICC criteria (11) were included and compared to 8 rheumatoid arthritis patients (disease control) and 10 normal controls. There was no difference between the three groups with respect to age $(59 \pm 18,51 \pm 19,52 \pm 15$, respectively) or sex, (women percent was $87 \%$ vs. $75 \%$ vs, $80 \%$ ). Disease duration was $5.2 \pm 3.3$ years. Seven SLE patients (18\%) suffered from active nephritis (mean $24 \mathrm{~h}$ proteinuria was $2900 \pm 210 \mathrm{mg} /$ day and mean serum creatinine level of $1.8 \pm 0.16 \mathrm{mg} / \mathrm{dl}$ ). Additional six patients $(16 \%)$, had been treated for lupus nephritis and achieved partial renal response (mean $24 \mathrm{~h}$ proteinuria was $750 \pm 190 \mathrm{mg} /$ day and mean serum creatinine level of $.08 \pm 0.10 \mathrm{mg} / \mathrm{dl}$ ). All other 25 SLE patients (66\%) were free of renal involvement (no proteinuria and serum creatinine was below $1.0 \mathrm{mg} / \mathrm{dl}$ ). All were ANA positive, and thirty patients were anti-dsDNA positive. Anti-cardiolipin antibodies IgG were detected in $13(34 \%)$ of patients. In patients with no renal involvement, the SLE disease activity score (SLEDAI) was recorded to be $8.2 \pm 2.7$, lower than in patients with renal involvement $12 \pm 3.3(p<0.03)$. Sixty six percent of patients were taking prednisone at a mean dose of $5 \pm 5.4 \mathrm{mg}$ per day. Other treatments included hydroxychloroquine (66\%), belimumab (33\%), azathioprine (26\%), mycophenolate mofetil (21\%), rituximab (16\%), methotrexate (16\%), abatacept (5\%), IVIG (5\%), (see table1).

\section{Sema3A urine levels}

Difference between the 3 groups (SLE patients, RA and healthy controls) studied with ANOVA variation was extremely significant $p=0.0006$. A further comparison between sema $3 \mathrm{~A}$ levels in each group demonstrated sema3A to be significantly lower in SLE patients compared to healthy controls and rheumatoid arthritis, $4.9 \pm 3.9 \mathrm{ng} / \mathrm{mL}, 8.5 \pm 2.7 \mathrm{ng} / \mathrm{mL}, 9.855 \pm 1.7 \mathrm{ng} / \mathrm{mL}, \mathrm{p}<0.05$ (figure $1 \mathrm{a}$ ). SLE patients with active nephritis or nephritis in partial remission $(n=13)$ demonstrated even lower urine sema3A 
concentration compared to those without renal involvement $4.0 \pm 3.4 \mathrm{ng} / \mathrm{ml}$ vs $6.5 \pm 3.8 \mathrm{ng} / \mathrm{ml}, \mathrm{p}=0.03$ (figure 1b). Sema3A inversely correlated with proteinuria $r=-0.43 p=0.01$ (figure 3) and SLEDAI 2K -0.3, $p=0.04$ (figure 2), but not with serum creatinine concentration, disease duration, autoantibodies and complement levels.

\section{Discussion}

This is the first study to assess urine sema3A secretion in SLE patients. We have demonstrated a significant lower level of sema3A in the urine of lupus patients compared to RA and healthy controls and an inverse correlation to disease activity (SLEDAI 2K) and proteinuria (due to lupus nephritis). In contrast, former studies reported higher secretion of urine sema3A in patients with diabetic nephropathy or contrast-media induced injury. While SLE manifests classical immune-mediated inflammation, contrastmedia induced renal injury is related to ischemic damage. The normal function of kidney podocytes, collecting tubules and endothelial cells is physiologically maintained by a balance between sema3A and vascular endothelial growth factor (VEGF) expression and secretion. Thus, excess or lack of either protein may disrupt glomerular filtration barrier homeostasis (12). Tight regulation of sema3A expression and secretion is therefore required for the maintenance of normal glomerular function. The pathophysiology of increased sema3A secretion in ischemic renal injury has been studied. Increased sema3A secretion in urine and decreased VEGF receptor expression was shown to be associated with glomerular endothelial damage, nephropathy, and proteinuria in diabetic mice (7). In a former report exposure of podocytes to recombinant sema3A decreased mRNA expression of plexinA1, A2 and A3 (VEGF receptors) and induced a dose-response podocin downregulation (13). sema3A was reported to induce a 10-fold increase in podocyte apoptosis and to decrease significantly the activity of the Akt survival pathway (14). In a recent study, increased sema3A urinary secretion was demonstrated to be associated with increased phosphorylated mTOR proteins leading to renal tubular injury and nephrotoxicity (15). While these studies may be explain the role of increased urinary sema3A in ischemic nephropathy, immune-mediated inflammation may have a different pathophysiology. High expression of VEGF was reported to play role in the pathophysiology of lupus nephritis by inducing ongoing angiogenesis and endothelial inflammation in the glomeruli of SLE patients. Increased renal tubular VEGF expression is associated with advanced glomerular damage and fibrosis (16). One may speculate that this could occur in part because of low sema3A secretion and the subsequent lack of its effect in counterbalancing and neutralizing that of VEGF.

We suggest several possible explanations to our finding of decreased urinary secretion of sema3a in SLE: 1. Local immune-mediated inflammation of endothelial cells and of tubuli in kidneys of SLE patients is responsible for the lower sema3A production and secretion. 2. Treg cells are one of the important sources for sema3A secretion. The density of FoxP3 + Treg cells was found to be decreased in kidney biopsies of patients with tubule-interstitial nephritis (17). Reduced expression of Treg cells in the glomeruli of SLE patients may result in losing one of the important sources of sema3A. 3. Low serum sema3A levels were previously reported to be correlated with SLE disease activity, and thus may account for the decreased secretion by the kidneys of these patients (5). Other mechanisms by which sema3A maintain 
glomerular/tubular normal function, and the physiological amount of urine sema3A that is required for this function are remaining to be determined. As noted above, sema3A is required for the suppression of ongoing angiogenesis, by inhibiting VEGF activity and the activation of inhibitory intracellular pathways that inhibit VEGF signal transduction (18). Sema3A has also been shown to be a regulator of glomerular vascular development. Deletion of sema3A resulted in defects in renal vasculature, excess endothelial cells within glomerular capillaries and the development of proteinuria. This suggests that balanced sema3A dosage is required for the maintenance of a normal filtration barrier (19).

In summary: The regulatory role of sema3A in SLE and other immune-mediated diseases is well established in many studies. This was confirmed by studies showing its beneficial therapeutic effects in animal models of SLE and asthma. The present study is the first to describe low urine sema3A in SLE patients compared to controls, and in association with lupus nephritis. The limitation of this study is the relatively small number of recruited patients (mainly the small number of patients suffering from lupus nephritis), which did not permit the assessment of many important SLE parameters. Future studies with larger cohorts are needed to assess the use of urine sema3A as a potential marker for renal involvement in SLE.

\section{Declarations}

Funding - No funding was received

Conflicts of interest/Competing interests - No conflict of interests to be declared.

Availability of data and material - All data available are presented herein the text of the manuscript.

Code availability - NA

Authors' contributions - All authors contributed to the manuscript, reviewed and approved it as presented here.

Ethics approval Ethics committee approval have been received

Consent to participate All patients have signed an informed consent form.

Consent for publication - We hereby transfer full rights for publication of this manuscript to Inflammation Journal. The manuscript has not been published elsewhere.

\section{References}

1. Neufeld, G., Y. Mumblat, and T. Smolkin, et al. 2016. The role of the semaphorins in cancer. Cell Adh Migr 10: 652-674.

2. Nishide, M., and A. Kumanogoh. 2018. The role of semaphorins in immune responses and autoimmune rheumatic diseases. Nat Rev Rheumatol 14: 19-31. 
3. Catalano, A. 2010. The neuroimmune semaphorin3A reduces inflammation and progression of experimental autoimmune arthritis. J Immunol 185: 6373-6383.

4. Cozacov, R., K. Halasz, T. Haj, and Z. Vadasz. 2017. Semaphorin3A: Is a key player in the pathogenesis of asthma. Clin Immunol 184: 70-72.

5. Vadasz, Z., T. Hai, and K. Halasz, et al. 2012. Semaphorin3A is a marker for disease activity and a potential immunoregulator in systemic lupus erythematosus. Arthritis Res Ther 14: R146.

6. Bejar, J., O. Kessler, A. D. Sabag, E. Sabo, O. B. Itzhak, and G. Neufeld, et al. Semaphorin3A: A potential therapeutic tool for lupus nephritis. Front Immunol 2018; (: 634.

7. Tufro, A. 2014. Semaphorin3A signaling, podocyte shape, and glomerular disease. Pediatr Nephrol 29: $751-755$.

8. Mohamed, R., P. Ranganathan, and C. Jayakumar, et al. 2014. Urinary semaphorin3A correlates with diabetic proteinuria and mediates diabetic nephropathy and associated inflammation in mice. $\mathrm{J} \mathrm{Mol}$ Med (Berl) 92: 1245-1256.

9. Ning, L., Z. Li, and D. Wei, et al. 2018. Urinary semaphorin3A as an early biomarker to predict contrast-induced acute kidney injury in patients undergoing percutaneous coronary intervention. Braz J Med Biol Res 51: e6487.

10. Gladman, D. D., D. Ibanez, and M. B. Urowitz. 2002. Systemic lupus erythematosus disease activity index 2000. J Rheumatol 29: 288-291.

11. Petri, M., A. M. Orbai, and G. S. Alarcón, et al. 2012. Derivation and validation of Systemic Lupus International Collaborating Clinics (SLICC) classification criteria for systemic lupus erythematosus. Arthritis Rheum 64: 2677-2686.

12. Aggarwal PK, Veron D, Thomas DB, et al. Semaphorin3a promotes advanced diabetic nephropathy. Diabetes. 2015;64(5):1743-59.

13. Tapia, R., F. Guan, I. Gershin, J. Teichman, G. Villegas, and A. Tufro. 2008. Semaphorin3a disrupts podocyte foot processes causing acute proteinuria. Kidney Int 73 (6): 733-740.

14. Guan, F., G. Villegas, J. Teichman, P. Mundel, and A. Tufro. 2006. Autocrine class 3 semaphorin system regulates slit diaphragm proteins and podocyte survival. Kidney Int 69: 1564-1569.

15. Song, M. F., Y. Yang, and Z. W. Yi, et al. 2018. Sema3A as a biomarker of the activated mTOR pathway during hexavalent chromium-induced acute kidney injury. Toxicol Lett 15: 226-235.

16. Avihingsanon, Y., T. Benjachat, A. Tassanarong, P. Sodsai, V. Kittikovit, and N. Hirankarn. 2009. Decreased renal expression of vascular endothelial growth factor in lupus nephritis is associated with worse prognosis. Kidney Int 75 (12): 1340-1348.

17. Rytkonen, S. H., P. Kulmala, and H. Autio-Harmainen, et al. 2018. FoxP3 + T cells are present in kidney biopsy samples in children with tubulointestinal nephritis and uveitis syndrome. Pdiatr Nephro/33: 287-293.

18. Hakroush, S., M. J. Moeller, and F. Theilig, et al. 2009. Effects of increased renal tubular vascular endothelial growth factor (VEGF) on fibrosis, cyst formation, and glomerular disease. Am J Pathol 
175: 1883-1895.

19. Reidy, K. J., G. Villegas, and J. Teichman, et al. 2009. Semaphorin3A regulates endothelial cell number and podocyte differentiation during glomerular development. Development 136: 3979-3989.

\section{Figures}
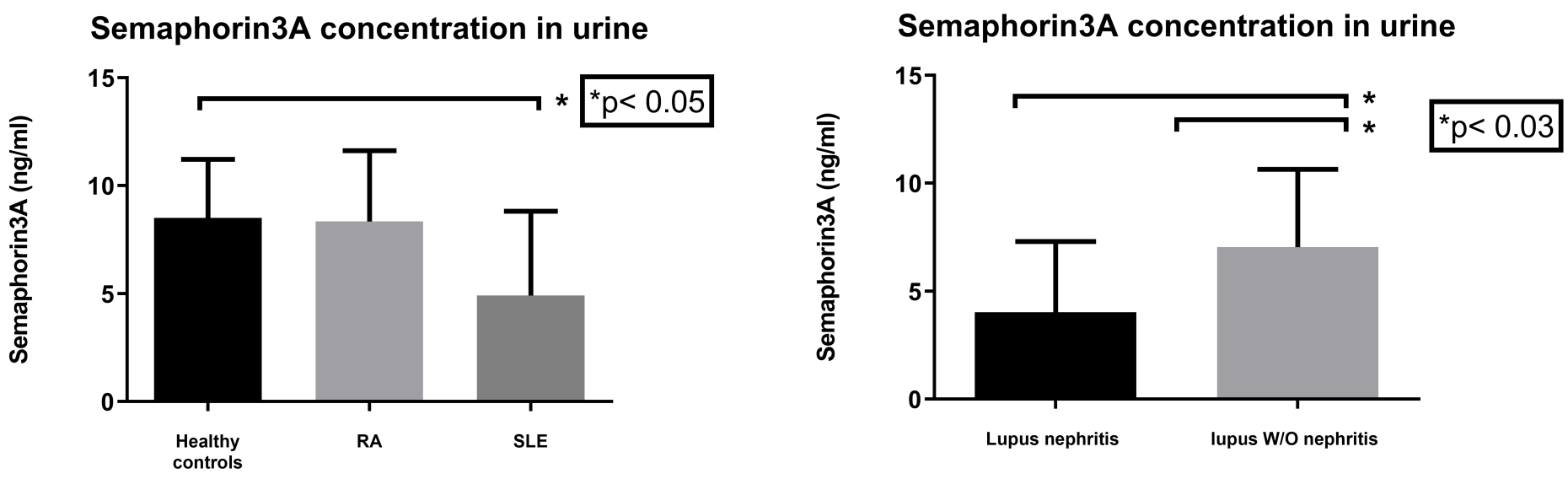

\section{Figure 1}

A further comparison between sema $3 \mathrm{~A}$ levels in each group demonstrated sema3A to be significantly lower in SLE patients compared to healthy controls and rheumatoid arthritis, $4.9 \pm 3.9 \mathrm{ng} / \mathrm{mL}$,

$8.5 \pm 2.7 \mathrm{ng} / \mathrm{mL}, 9.855 \pm 1.7 \mathrm{ng} / \mathrm{mL}, \mathrm{p}<0.05$ (figure a). SLE patients with active nephritis or nephritis in partial remission $(n=13)$ demonstrated even lower urine sema3A concentration compared to those without renal involvement $4.0 \pm 3.4 \mathrm{ng} / \mathrm{ml}$ vs $6.5 \pm 3.8 \mathrm{ng} / \mathrm{ml}, \mathrm{p}=0.03$ (figure b). 


\section{Correlation of urine semaphorin3A with SLEDAI2K}

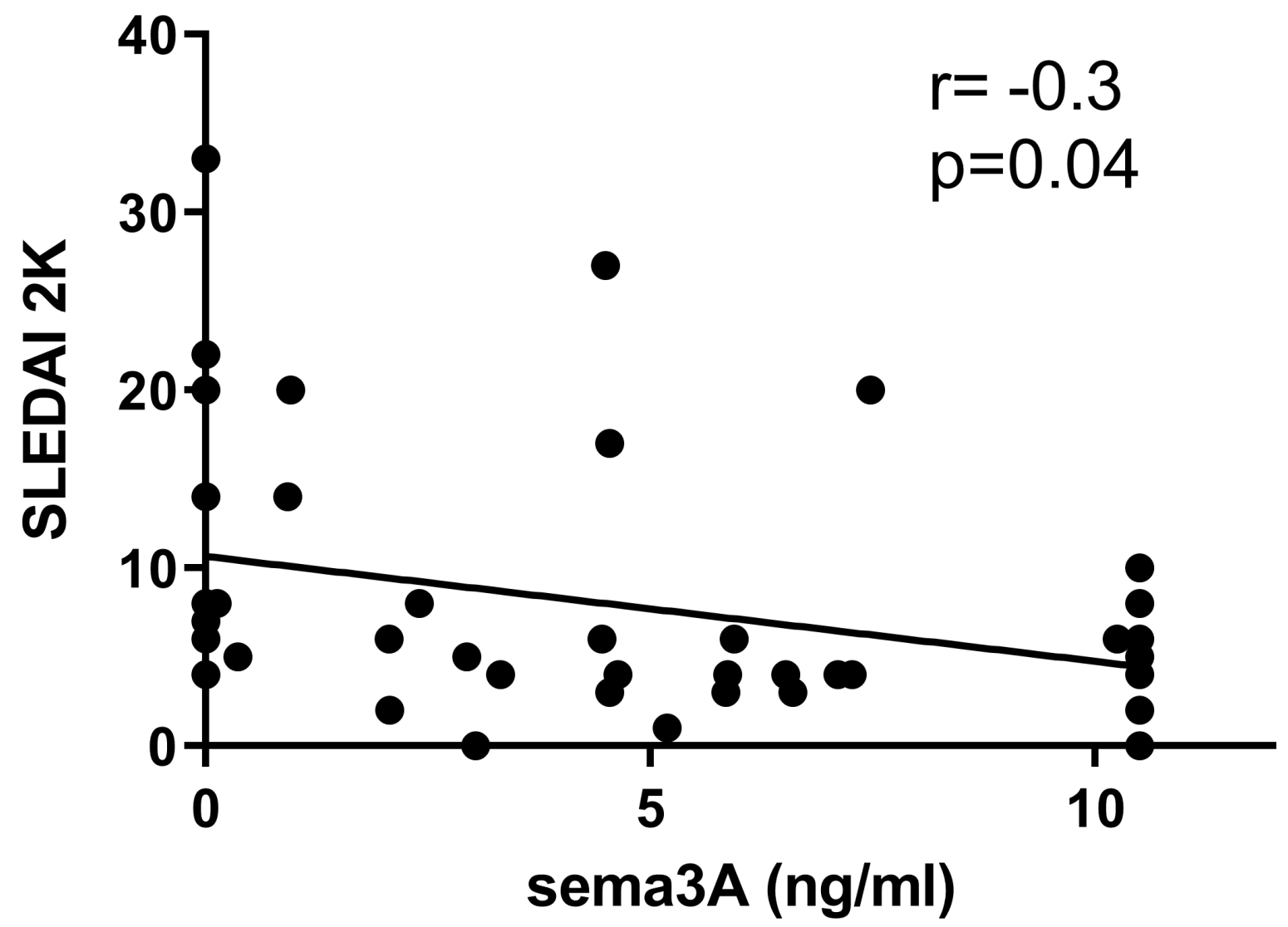

Figure 2

Sema3A inversely correlated with proteinuria $r=-0.43 p=0.01$ and SLEDAI $2 \mathrm{~K}-0.3, p=0.04$, but not with serum creatinine concentration, disease duration, autoantibodies and complement levels. 
Correlation between urine semaphorin $3 \mathrm{~A}$ and proteinuria

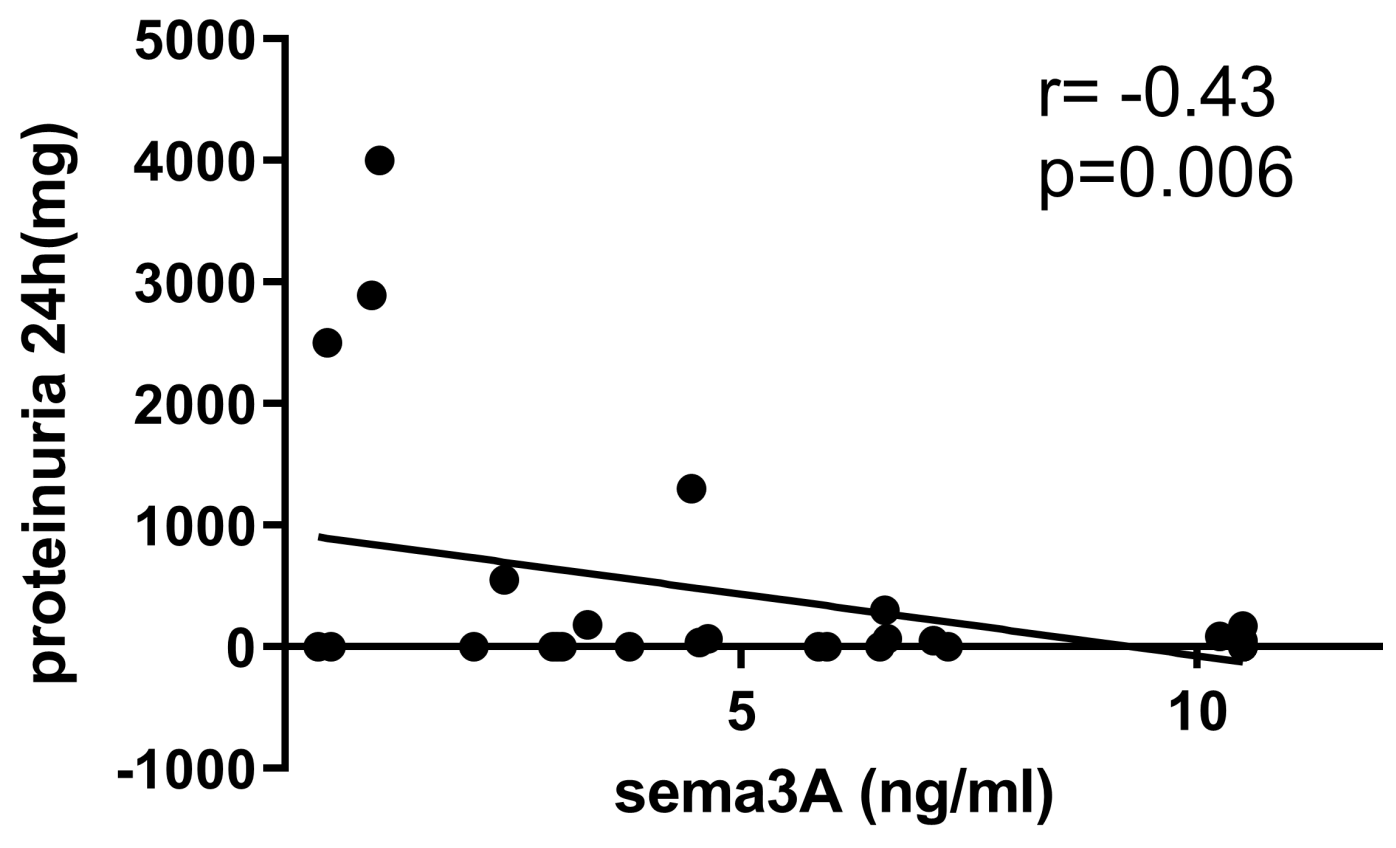

Figure 3

Sema3A inversely correlated with proteinuria $r=-0.43 p=0.01$ 